\title{
POLYNOMIAL APPROXIMATIONS ON A POLYDISC ${ }^{1}$
}

\author{
CHARLES K. CHUI \\ (Received 15 January, revised 30 March 1971)
}

Communicated by J. B. Miller

\section{Introduction and Results}

Throughout this paper, we will use the terminologies and notations as in [4]. Thus, $U^{N}$ denotes the open unit polydisc in the space $\mathbb{C}^{N}$ of $N$ complex variables, $T^{N}$ the distinguished boundary of $U^{N}$ and

$$
V^{N}=\left\{\left(z_{1}, \cdots, z_{N}\right) \in \mathbb{C}^{N}:\left|z_{j}\right|>1 \text { for } j=1, \cdots, N\right\} .
$$

We say that $\boldsymbol{n}=\left(n_{1}, \cdots, n_{N}\right)$ tends to infinity if $n_{j} \rightarrow \infty$ for each $j=1, \cdots, N$. A polynomial $P$ of $N$ complex variables $\left(z_{1}, \cdots, z_{N}\right)$ is said to be of order $\boldsymbol{n}$ $=\left(n_{1}, \cdots, n_{N}\right)$ if for each $j, 1 \leqq j \leqq N,\left(\partial^{k} / \partial z_{j}^{k}\right) P\left(z_{1}, \cdots, z_{N}\right)$ is not identically zero for $k=n_{j}$ but is the zero function for each $k>n_{j}$. Let $P$ be a polynomial in $\mathbb{C}^{N}$. If the only zeros of $P$ in $\bar{U}^{N} \cup \bar{V}^{N}$ lie on $T^{N}$, then $P$ will be called a $T^{N}$ polynomial. Hence, for $N=1, T=T^{1}$, a $T$-polynomial is a polynomial such that all its zeros lie on the unit circle $T$. In the case of one complex variable, different kinds of $T$-polynomial approximation theorems were obtained in $[1,2$, and 3]. In this note, we establish these theorems for any $N \geqq 1$.

THEOREM 1. If $f$ is holomorphic and does not vanish in $U^{N}$, there exist $T^{N}$-polynomials $Q_{m}$ which converge to funiformly on every compact subset of $U^{N}$.

THEOREM 2. If $f \in H^{p}=H^{p}\left(U^{N}\right)$, where $1 \leqq p \leqq \infty$, and does not vanish in $U^{N}$, there exist $T^{N}$-polynomials $Q_{m}$ which converge to $f$ uniformly on every compact subset of $U^{N}$ and satisfy $\left\|Q_{m}\right\|_{p} \leqq 2\|f\|_{p}$ for all $\boldsymbol{m}$.

Here, uniform convergence on compact subsets of $U^{N}$ cannot be replaced by convergence in $H^{p}$. For $p=\infty$, it is clear, and for $1 \leqq p<\infty$, it is proved for $N=1$ in [2].

Let $\mathscr{H}^{p}=\mathscr{H}^{p}\left(U^{N}\right)(1 \leqq p<\infty)$ be the class of all holomorphic functions $f$ in $U^{N}$ such that

1 Research supported by NSF Grant GP-12042. 


$$
\mathbf{U}_{\mathbf{D}_{p}}=\left\{1 / \pi^{N} \int_{U^{N}}|f|^{p}\right\}^{1 / p}<\infty .
$$

It is clear that each $\mathscr{H}^{p}$ with the norm $\|_{p}$ is a Banach space. For the spaces $\mathscr{H}^{p}$, we have a stronger result.

THEOREM 3. If $f \in \mathscr{H}^{p}(1 \leqq p<\infty)$ and does not vanish in $U^{N}$, there exist $T^{N}$-polynomials $Q_{m}$ such that

$$
Q_{m}-f \mathbf{M}_{p} \rightarrow 0
$$

The author wishes to thank the referee for his helpful suggestions.

\section{Proofs of the above theorems}

For $z=\left(z_{1}, \cdots, z_{N}\right)$ where $z_{j} \neq 0, j=1, \cdots, N$, we use the notation $1 / z$ $=\left(1 / z_{1}, \cdots, 1 / z_{N}\right)$. Let $P$ be a polynomial in $\mathbb{C}^{N}$ with no zero in $\bar{U}^{N}$ and let $M(z)=z_{1}^{n_{1}}, \cdots, z_{N}^{n_{1}}$ be a monomial of sufficiently large order so that

$$
Q(z)=P(z)+M(z) \tilde{P}(1 / z)
$$

is a polynomial. Here, $\tilde{P}$ is the polynomial whose coefficients are the complex conjugates of the coefficients of $P[\mathrm{cf} .4]$. Then $\overline{P(w)}=\widetilde{P}(1 / w)$ for all $w \in T^{\mathrm{V}}$. Hence,

$$
|M(z) \tilde{P}(1 / z) / P(z)|=1
$$

for each $z$ on $T^{N}$. Since $P$ has no zero in $\bar{U}^{N}$, by the maximum principle, we conclude from (1) and (2) that $Q$ has no zero in $\bar{U}^{N}$, except possibly on $T^{N}$. Now, since $M(z) \tilde{M}(1 / z)=1$, we have

$$
M(z) \widetilde{Q}(1 / z)=Q(z) .
$$

Hence, $\widetilde{Q}(1 / z)$ does not vanish in $\bar{U}^{N}$, except possibly on $T^{N}$. That is, $\tilde{Q}(z)$, and hence $Q(z)$, has no zero in $\bar{V}^{N}$, except possibly on $T^{N}$. Therefore, $Q$ is a $T^{N}$ polynomial.

Now, let $f$ be holomorphic in $U^{N}$ and $f(z) \neq 0$ for all $z$ in $U^{N}$. Then for each $r, 0<r<1$, the function $f_{r}$ defined by $f_{r}(z)=f(r z)$, where $r z=\left(r z_{1}, \cdots, r z_{N}\right)$, is holomorphic and does not vanish in $(1 / r) U^{N}$, and can then be uniformly approximated on $\bar{U}^{N}$ by polynomials which do not vanish in $\bar{U}^{N}$. But $f_{r} \rightarrow f$ uniformly on each compact subset of $U^{N}$ as $r_{i}^{\wedge}$. Hence, $f$ can be approximated uniformly on each compact subset of $U^{N}$ by polynomials $P_{n}$ which do not vanish on $\bar{U}^{N}$. Let

$$
Q_{m, n}(z)=P_{n}(z)+M_{m}(z) \tilde{P}_{n}(1 / z)
$$

where $M_{m}$ are monomials of sufficiently large order $\boldsymbol{m}$. By (2) and the maximum principle, we see that 


$$
\left|M_{m}(z) \tilde{P}_{n}(1 / z)\right| \leqq\left|P_{n}(z)\right|
$$

in $\bar{U}^{N}$ for all sufficiently large $\boldsymbol{m}$. Since $P_{n} \rightarrow f$ uniformly on compact subsets of $U^{N}, M_{m}(z) \tilde{P}_{n}(1 / z) \rightarrow 0$ on compact subsets of $U^{N}$ as $\boldsymbol{n}$ and suitable $\boldsymbol{m}=\boldsymbol{m}(\boldsymbol{n})$ tend to infinity. That is, a sequence of $T^{N}$-polynomials can be chosen from $Q_{m, u}$ to approximate $f$ uniformly on every compact subset of $U^{N}$. This proves the first theorem. If, in addition, $f$ is in $H^{p}(1 \leqq p \leqq \infty)$, we can choose the $P_{n}$ so that $\left\|P_{n}\right\|_{p} \leqq\|f\|_{p}$ for all $n$. Hence, using (4) and (5), we have $\left\|Q_{m, n}\right\|_{p} \leqq 2\|f\|_{p}$ for all $\boldsymbol{n}$ and all sufficiently large $\boldsymbol{m}$, proving Theorem 2 . Now, suppose that $f \in \mathscr{H}^{p}(1 \leqq p<\infty)$ and does not vanish in $U^{N}$. We can choose the $P_{n}$, which do not vanish in $\bar{U}^{N}$, such that $P_{n}-f \mathbf{\bigsqcup}_{p} \rightarrow 0$. For each $r, 0<r<1$, let $K_{r}=r \bar{U}_{N}$ and let $D_{r}$ be the complement of $K_{r}$ with respect to $U^{N}$. Since $f \in \mathscr{H}^{p}$ and the (2N-dimensional) Lebesgue measure of $D_{r}$ tends to zero as $r \uparrow 1$, we have

$$
\lim _{r \uparrow 1} \int_{D_{r}}|f|^{p}=0
$$

Hence, for each $\varepsilon>0$, we can choose $1-r>0$ so small that

$$
\int_{D_{r}}\left|P_{n}\right|^{p}<\varepsilon
$$

for all large $\boldsymbol{n}$. Now, for all sufficiently large $\boldsymbol{m}$, we obtain, using (5),

$$
\| M_{m}(z) \widetilde{P}_{n}(1 / z) \mathbf{\coprod}_{p} \leqq \max _{K_{r}}\left|M_{m}(z) \tilde{P}_{n}(1 / z)\right|+\left\{\left.\frac{1}{\pi^{N}} \int_{D_{r}}\left|P_{n}\right|\right|^{p}\right\}^{1 / p} .
$$

Again, since $P_{n} \rightarrow f$ uniformly on $K_{r}$, the $\boldsymbol{m}=\boldsymbol{m}(\boldsymbol{n})$ can be chosen such that $M_{\boldsymbol{m}}(z) \widetilde{P}_{\boldsymbol{n}}(1 / z) \boldsymbol{U}_{p} \rightarrow 0$ as $\boldsymbol{n}$ and $\boldsymbol{m}$ tend to infinity. Hence, a sequence of $T_{N^{-}}$ polynomials $Q_{m}$ can be chosen from the $Q_{m, n}$ such that $\backslash Q_{m}-f \mathbf{\bigsqcup}_{p} \rightarrow 0$. This completes the proof of the third theorem.

\section{References}

[1] C. K. Chui, 'Bounded approximation by polynomials whose zeros lie on a circle', Trans. Amer. Math. Soc. 138 (1969), 171-182.

[2] C. K. Chui, 'C-polynomial approximation of $H^{p}$ and $\mathscr{H}^{p}$ functions', J. Math. Analysis and Appl. To appear.

[3] J. Korevaar, 'Approximation by polynomials whose zeros lie on a circle', Nieuw Arch. Wisk (3) 10 (1962), 11-16.

[4] W. Rudin, Function theory in polydiscs (W. A. Benjamin, New York, 1969).

Texas A\&M University

College Station, Texas 77843, U.S.A. 